\title{
Randomized Trial of Group Therapy for Repeated Deliberate Self-Harm in Adolescents
}

\author{
ALISON WOOD, M.R.C.PSYCH., GEMMA TRAINOR, M.A., JUSTINE ROTHWELL, B.A., ANN MOORE, B.A., \\ AND RICHARD HARRINGTON, M.D.
}

\begin{abstract}
Objective: To compare group therapy with routine care in adolescents who had deliberately harmed themselves on at least two occasions within a year. Method: Single-blind pilot study with two randomized parallel groups that took place in Manchester, England. Sixty-three adolescents aged 12 through 16 years were randomly assigned to group therapy and routine care or routine care alone. Outcome data on suicide attempts were obtained without knowledge of treatment allocation on all randomized cases ( $62 / 63$ by direct interview) on average 29 weeks later. The primary outcomes were depression and suicidal behavior. Results: In intention-to-treat analyses, adolescents who had group therapy were less likely to be "repeaters" at the end of the study (i.e., to have repeated deliberate self-harm on two or more further occasions) than adolescents who had routine care (2/32 versus $10 / 31$; odds ratio 6.3 ), but the confidence intervals for this ratio were wide (95\% confidence interval 1.4 to 28.7 ). They were also less likely to use routine care, had better school attendance, and had a lower rate of behavioral disorder than adolescents given routine care alone. The interventions did not differ, however, in their effects on depression or global outcome. Conclusions: Group therapy shows promise as a treatment for adolescents who repeatedly harm themselves, but larger studies are required to assess more accurately the efficacy of this intervention. J. Am. Acad. Child Adolesc. Psychiatry, 2001, 40(11):1246-1253. Key Words: therapy, attempted suicide, repetition, sexual abuse, major depression.
\end{abstract}

Repeated deliberate self-harm is an important problem among adolescents for four main reasons. First, it is strongly associated with recurrent psychosocial problems, such as sexual abuse (Brown et al., 1999) and depression (Hawton et al., 1999b), which are strong predictors of poor outcomes (Fergusson et al., 1996; Harrington et al., 1990). Second, it places considerable demands on services, espe-

Accepted May 29, 2001.

From the Department of Child and Adolescent Psychiatry, Withington Hospital, South Manchester, England (Dr. Wood and Miss Trainor) and the University Department of Child Psychiatry, Royal Manchester Children's Hospital, Pendlebury, Manchester, England (Miss Rothwell, Mrs. Moore, and Professor Harrington).

This paper is dedicated to the memory of Ann Moore, whose ideas and endurance contributed so much to many research projects.

This research was supported by a project grant from the Mental Health Foundation and by a Training Fellowship to Miss Trainor from the National Health Service Executive North West. The authors thank Daphne Kounali of the Department of Medical Statistics at Hope Hospital, who conducted the randomization, and Dr. Adrian Sutton, consultant child and adolescent psychiatrist, for his help in supervising the groups.

Address correspondence and requests for the manual to Dr. Harrington, University Department of Child Psychiatry, Royal Manchester Children's Hospital, Pendlebury, Manchester M274HA,England; e-mail: r.c.harrington@man.ac.uk.

0890-8567/01/4011-1246@2001 by the American Academy of Child and Adolescent Psychiatry. cially expensive services such as residential units. Third, in some geographical areas the proportion of adolescent suicide attempters who repeat is increasing (Hawton et al., 2000). Finally, and most importantly, it can be followed by completed suicide (Hawton et al., 1999a).

Despite the importance of repeated deliberate selfharm among adolescents, very little is known about how it should best be managed. Indeed, a recent systematic review (NHS Centre for Reviews and Dissemination, 1998) found only two randomized studies of interventions for young people aged less than 17 years, neither of which was primarily concerned with young people who had deliberately harmed themselves on several occasions.

In line with these considerations, this article describes a randomized trial of a group intervention for adolescents who repeatedly harmed themselves. The main hypothesis was that in comparison with routine care alone, the addition of a group intervention to routine care would lead to lower levels of depression and a lower risk of deliberate self-harm. Depression was included as a primary outcome because it is very prevalent among self-harm cases and predicts response to treatment (Harrington et al., 1998). 


\section{METHOD}

\section{Entry Criteria}

Participants were eligible if they (1) were aged 12 to 16 years, (2) had been referred to the child and adolescent mental health service of a health district in South Manchester, England, following an incident of deliberate self-harm, and (3) reported that they had deliberately harmed themselves on at least one other occasion during the previous year. The term deliberate self-harm was defined as "any intentional self-inflicted injury, irrespective of the apparent purpose of the act" (Hawton and Catalan, 1982). The most common forms of deliberate self-harm in this study were deliberate self-poisoning and deliberate self-cutting. Deliberate self-poisoning was defined as "the deliberate ingestion of more than the prescribed amount of medical substances, or ingestion of substances never intended for human consumption, irrespective of whether harm was intended" (Hawton and Catalan, 1982). We excluded from this definition accidental overdoses of drugs usually taken for kicks and any overdose of alcohol.

Participants were deemed ineligible if (1) a senior child and adolescent psychiatrist judged that they were too suicidal for ambulatory care, (2) their current situation meant they could not attend the groups (e.g., incarcerated), (3) they suffered from a psychotic disorder, or (4) it was unlikely they could benefit from a group intervention (e.g., learning problems).

\section{Interventions and Therapists}

When the intervention was first developed, a group format (as opposed to an individual approach) was chosen for two main reasons, the first clinical and the second administrative. From a clinical perspective, groups were felt to be a good setting for dealing with some of the problems that are especially prevalent among suicidal adolescents, such as poor peer relationships and impaired problem-solving. From an administrative perspective, it was envisaged that a group would (1) reduce the need for other forms of care, such as individual therapy, and (2) form the focus for the development of a team who could become specialized in dealing with deliberate self-harm. These administrative issues are important in the United Kingdom because rates of deliberate self-harm in Britain are among the highest in Europe (Hawton et al., 1998) and British child mental health professionals therefore spend much time in the aftercare of these vulnerable adolescents (Kerfoot and Huxley, 1995).

The group therapy, termed developmental group psychotherapy, was specifically designed for adolescents who harm themselves and has been described in a manual (available from the corresponding author free of charge via e-mail). The therapy is "developmental" in that it was designed to meet the needs of adolescents and has a focus on the adolescent growing through difficulties by using positive corrective therapeutic relationships. It brings together techniques from a variety of other therapies, including the problem-solving and cognitive-behavioral interventions that we have previously used with depressed or suicidal adolescents and their families (Harrington et al., 1998; Kroll et al., 1996; Wood et al., 1996), dialectical behavior therapy (Linehan et al., 1991), and psychodynamic group psychotherapy. The intervention comprises an initial assessment phase, attendance at six "acute" group sessions, followed by weekly group therapy in a "long-term group," which can continue until the young person feels ready to leave. The "acute" group program is orientated around six themes, which previous research suggests are important in adolescents who harm themselves (Hawton et al., 1999b; Kerfoot, 1988; Kerfoot et al., 1996). These themes are relationships, school problems and peer relationships, family problems, anger management, depression and self-harm, and hopeless- ness and feelings about the future. The "long-term group" is facilitated by the same therapists, but it places much more emphasis on group processes. In this study it was supervised by an experienced psychodynamic group therapist. Both the acute group and the long-term group run continuously, and adolescents can join them at any time (the groups were designed in this way because adolescents who repeatedly harm themselves usually present in crisis and often cannot wait for several weeks before receiving treatment).

Two therapists, a senior nurse and a psychiatrist, administered all of the sessions. One of them (G.T.) pilot-studied the therapy for her master's degree, and the other (A.W.) had much previous experience with cognitive-behavioral therapy (Wood et al., 1996). Group sessions were sometimes augmented by individual sessions, which were given by the same therapists.

Routine care was monitored with a resource use questionnaire developed in a previous study by our group (Byford et al., 1999). It consisted of a variety of interventions given by community psychiatric nurses and psychologists, which included family sessions and nonspecific counseling with the adolescent. It also included psychotropic medication, when clinically indicated.

\section{Procedures for Assignment and Masking}

The unit of randomization was the adolescent. After written consent was obtained, the clinician telephoned an independent statistician at a distant site (D.K.) who assigned a trial number and then randomly allocated participants to the group or routine care. Adolescents joined the groups as soon as they were randomized. Treatment allocation was concealed from the outcome assessors, who were asked at the end of the study which treatment had been given to which adolescent. Their responses were no better than chance (34/63 correct).

Because studies of psychosocial treatments can only ever be singleblind, participant expectancies of treatment could bias the results. The adolescents' expectation of treatment results after randomization was therefore assessed by using a 0 to 8 continuous scale (Marks, 1981), in which 8 was a very high expectation that treatment would help and 0 that it would not help at all. Adolescents in the group therapy arm had a similar level of expectation (mean $=4.6,95 \%$ confidence interval [CI 4.0 to 5.3 ) to those in the routine care arm (mean $=4.9,95 \% \mathrm{CI}$ 3.8 to 6.0$)$

\section{Assessment of Effectiveness}

Measures were completed before treatment and at 6 weeks and 7 months after randomization. The primary outcomes were self-reported depressive symptoms (Angold et al., 1987; Wood et al., 1995), suicidal thinking (Reynolds, 1988), and repetition of self-harm assessed by portions of an interview-based assessment of suicidal behavior (Kerfoot, 1984; Kerfoot and McHugh, 1992). Secondary outcomes included depressive disorder and behavioral problems rated with the Schedule for Affective Disorders and Schizophrenia for School-Age Children (KSADS) (Ambrosini, 2000; Puig-Antich and Chambers, 1978) and diagnosed using the DSM-IV (American Psychiatric Association, 1994). Several versions of the K-SADS are available. We used the one developed by Kaufman and colleagues (1996).

Global outcome was assessed with the Health of the Nation Outcome Scales for Children and Adolescents (HoNOSCA) (Gowers et al., 1999a). The HoNOSCA measures a broad range of outcomes, including symptoms and psychosocial impairment. It has 13 subscales, each rated from 0 (no problems) through 4 (severe problems). The subscales include emotional symptoms, behavioral problems, substance abuse, school attendance problems, and peer relationships. It was developed as a generic outcome measure for child and adoles- 
cent mental health services and is of known reliability and validity in U.K. clinical samples (Garralda et al., 2000; Gowers et al., 1999b).

Interview data (i.e., K-SADS, suicidal behavior, and the HoNOSCA) were obtained from the adolescent, corroborated from other sources, and combined by the assessor into a best estimate. In making this estimate, precedence was given to a rating when it was based on a clear description of a symptom.

\section{Assessment of Demographic and Background Characteristics}

Demographics were assessed with the K-SADS. Psychosocial adversity was assessed by reviewing the charts and other records. Psychosocial stresses were coded with the International Classification of Diseases, Axis V scheme (World Health Organization, 1996). Abuse was considered "definite" either when it had led to some kind of court order (e.g., the child being taken into the care of social services) or when an adult was convicted of the abuse. Abuse was considered "probable" when the charts or other records contained a clear statement by the child or other professional that abuse had occurred.

\section{Sample Size Projection}

We projected a sample size of approximately 25 cases in each group because in a previous study (Wood et al., 1996) we had found significant effects of a cognitive-behavioral intervention on depression with that sample size. When this study was devised and funded, however, it was acknowledged that it could detect only a large treatment effect on repetition of deliberate self-harm. Indeed, in the protocol we estimated that with that sample size we would have only a $70 \%$ chance of detecting a large difference in repetition risk $(20 \%$ in the intervention group and $50 \%$ in the control group), using a log-rank survival test (Machin and Campbell, 1987).

\section{Statistical Analysis}

This pragmatic trial set out to measure effectiveness, that is the benefit the treatment produces in routine clinical practice. Pragmatic trials are concerned with the total differences between two treatments, including active effects of the treatment and the lack of effects that might result from poor compliance, as this will best reflect the likely overall response in clinical practice. Therefore all analyses were conducted by "intention to treat." There are many different definitions of intention to treat (Hollis and Campbell, 1999), but in this study we used the most stringent in which all randomized cases were included, regardless of whether they started or completed treatment. The analysis was conducted just once, all cases were analyzed as allocated, and no interim or subgroup analyses were permitted. The data were analyzed with SPSS 9.0 for Windows. For continuous variables changes from baseline were calculated for the outcomes, and $t$ tests for independent samples were used to compare the two arms of the trial. For time-varying outcomes (e.g., time since randomization to the first or second episode of deliberate self-harm) differences between the arms were tested in a Cox proportional hazards model, with the number of episodes of deliberate self-harm before randomization as a covariate. Differences between the groups on continuous variables that were not normally distributed were examined with the Mann-Whitney test.

To convey the clinical significance of treatment effects on categorical outcomes, the "number needed to treat" was calculated as 1 divided by the absolute risk ratio. This is the number of patients who need to be treated to prevent one bad outcome. Ninety-five percent confidence intervals for this number were calculated with the method recommended by Sackett and colleagues (2000). Following convention, all numbers needed to treat were rounded up.

\section{RESULTS}

\section{Participant Flow and Follow-up}

Figure 1 shows the trial profile. During the study 83 eligible adolescents were seen by the service. Of these, there were three adolescents whose care situation meant they could not attend the groups (e.g., incarcerated in a secure social services residential facility) and one who was believed unlikely to benefit from the group because of learning disability. Sixteen potential participants refused the trial, or the groups, or both. The 20 nonparticipants were slightly older (mean age 15.0 years, SD 0.8 years) than the participants (mean age 14.2 years, SD 1.6 years), a significant difference $\left(t_{81}=2.0, p=.048\right)$. Otherwise, however, there were no significant differences between participants and nonparticipants in demographic and background features.

The remaining 63 adolescents were then randomly allocated to group therapy $(n=32)$ or routine care $(n=$ $31)$. Sixty-two $(98 \%)$ of 63 were interviewed on average 29 weeks later, and the mother of the adolescent who could not be interviewed provided information about

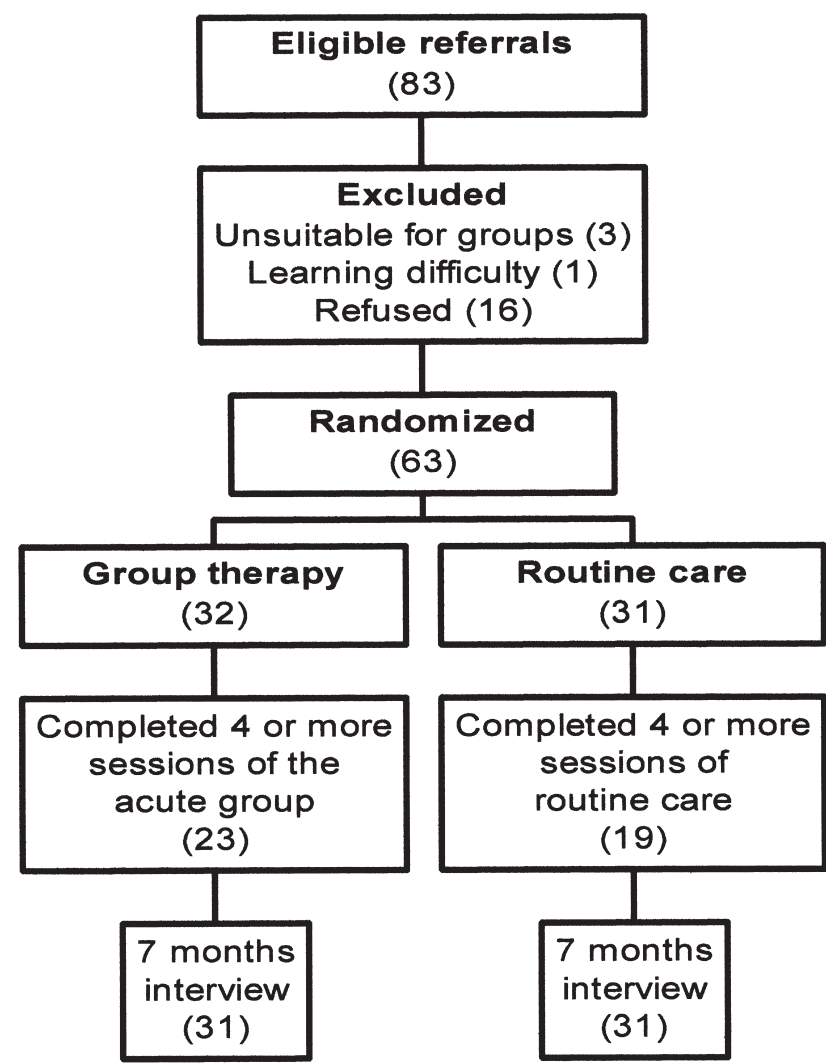

Fig. 1 Trial profile. 
her daughter's outcome. Duration of follow-up did not differ significantly between adolescents attending the groups (mean $=30.3$ weeks, $95 \%$ CI 28.2 to 32.5 ) and those having routine care $($ mean $=28.5$ weeks, $95 \% \mathrm{CI}$ 27.0 to 30.1 ).

\section{Participant Characteristics}

Table 1 shows the demographic and clinical characteristics of randomized adolescents, by treatment group. The two groups were well matched. Both groups had harmed themselves on average four times before the study. The most common methods of deliberate self-harm were taking overdoses and cutting. Eleven of 63 adolescents had also harmed themselves in other ways, which included burning, hanging, and strangulation. The adolescents tended to come from disadvantaged backgrounds, with the majority from families in which the main wage earner either was doing a manual job or was unemployed. Few were living with both parents. In approximately one half of the sample there was evidence of "definite" or "probable" sexual or physical abuse at some point during the young person's lifetime.

As Table 1 shows, many participants had major depressive disorders; this finding is in agreement with other research on adolescents who repeatedly harm themselves (Hawton et al., 1999b). There was also a high prevalence of behavioral disturbance. Many regularly used illicit drugs and approximately one half were intoxicated with alcohol at least weekly.

\section{Treatments}

Adolescents in group therapy attended a median of 8 group sessions (range $0-19$ sessions) over the 6 months of the trial. In addition, they had a median of 2.5 (range $0-10$ sessions) additional individual sessions given by the same therapists. The individual sessions were usually undertaken either when extra cognitive-behavioral work was needed that had to be tailored to the child's specific needs or when issues arose that could not easily be discussed in the group (e.g., matters related to sexual abuse).

Routine care was used much less for those in the group arm of the trial (median 1 session, range 0-25 sessions) than for those in the routine care arm (median 4 sessions, range $0-30$ sessions), a significant difference ( $U=253$, $p$ $<.01)$. Routine care comprised individual supportive sessions or supportive sessions with parents. Routine care was the same in the group arm of the trial as in the routine care arm, but there was less of it. Antidepressants were prescribed for 5 of 32 cases in the routine care arm, compared with 8 of 30 cases in the group therapy arm. One adolescent in the routine care arm was admitted to a psychiatric facility during the study.

\section{Repetition of Deliberate Self-Harm}

Adolescents allocated to the group tended to have fewer episodes of deliberate self-harm (mean $=0.6,95 \%$ CI 0.3 to 0.9 ) than adolescents allocated to routine care (mean $=1.8,95 \%$ CI 0.6 to 3.0). There was a particularly strong effect of the intervention on the risk of still being a "repeater" (two or more episodes of deliberate self-harm) at the end of the study. Thus, in a Cox regression model that included number of episodes of deliberate self-harm before randomization, the risk of being a repeater after randomization was significantly higher among adolescents allocated to routine care $(n=10 / 31$ or $32 \%)$ than among adolescents allocated to the group $(n=$ $2 / 32$ or $6 \%$ ), with an odds ratio of 6.3 (95\% CI 1.4 to 28.7). The mean time to first repetition was longer in the group arm of the trial (11.9 weeks, SD 7.2 weeks) than in the routine care arm (7 weeks, SD 6.3 weeks), giving a mean difference of 4.9 weeks (95\% CI 0.0 to 9.8), which was statistically significant $\left(t_{30}=2.1, p<.05\right)$.

\section{Depression, Suicidal Thinking, and Other Outcomes}

As Table 2 shows, there was no significant effect of the intervention on depressive symptoms or suicidal thinking. Neither did the groups differ with respect to global outcome, as assessed by the total score on the HoNOSCA. However, school attendance, measured by one of the subscales of the HoNOSCA, was significantly better at the end of the study in those having the group intervention than in controls $\left(\chi_{4}^{2}=10.4, p=.03\right)$.

Rates of most mental disorders diagnosed with the KSADS at the 7-month follow-up did not differ between the two arms of the trial. However, the rate of behavioral disorder (oppositional, conduct, or attention-deficit/ hyperactivity disorder) was lower in those who had group therapy (6/32 or 19\%) than in those who did not $(11 / 31$ or $35 \%)$. In a logistic regression that controlled for duration of follow-up and behavioral disorder at baseline, there was a treatment effect on behavioral disorder at 7 months (odds ratio $=0.3,95 \%$ CI 0.1 to 1.0 ).

\section{Relationship of Amount of Treatment and Repetition}

Adolescents who had more sessions of group therapy were less likely to repeat deliberate self-harm. Thus, in a 
WOOD ET AL.

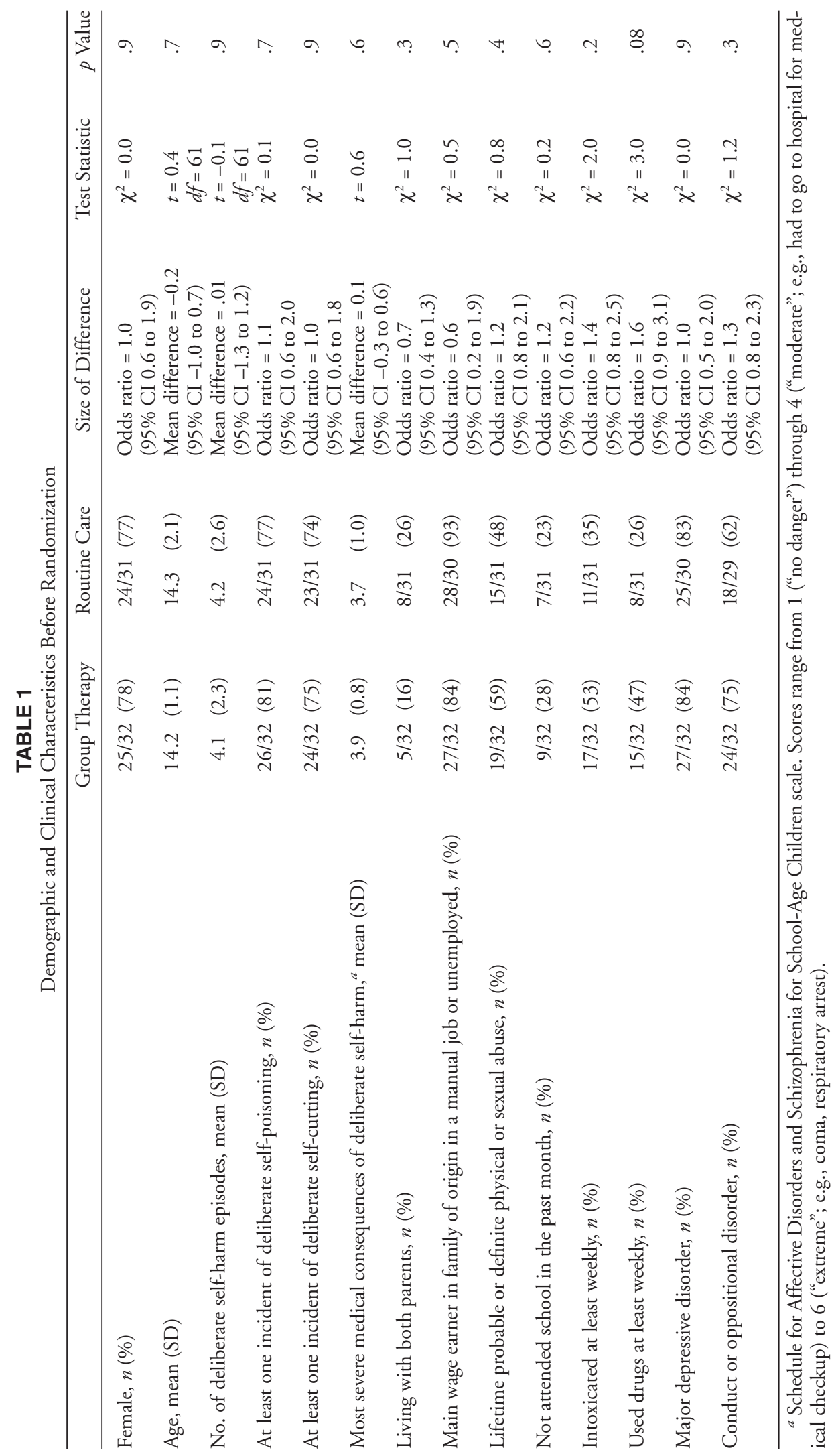


TABLE 2

Depression, Suicidal Thinking, and Global Adjustment

\begin{tabular}{|c|c|c|c|c|c|c|c|c|c|}
\hline & \multicolumn{3}{|c|}{ Baseline } & \multicolumn{3}{|c|}{7 Months } & \multicolumn{3}{|c|}{$\begin{array}{c}\text { Change From } \\
\text { Baseline to } 7 \text { Months }\end{array}$} \\
\hline & $n$ & Mean & $\mathrm{SD}$ & $n$ & Mean & $\mathrm{SD}$ & $n$ & Mean & SD \\
\hline \multicolumn{10}{|l|}{ Mood and Feelings Questionnaire ${ }^{a}$} \\
\hline Group therapy & 32 & 40.6 & 10.6 & 29 & 21.9 & 15.6 & 29 & 18.8 & 16.0 \\
\hline Routine care & 29 & 39.8 & 14.2 & 29 & 23.4 & 18.0 & 27 & 15.3 & 13.0 \\
\hline Mean difference & \multirow{2}{*}{\multicolumn{3}{|c|}{$\begin{array}{c}0.8 \\
(-0.6 \text { to } 7.2)\end{array}$}} & \multirow{2}{*}{\multicolumn{3}{|c|}{$\begin{array}{c}-1.5 \\
(-10.4 \text { to } 7.3)\end{array}$}} & & 3.5 & \\
\hline $95 \%$ CI of difference & & & & & & & \multicolumn{3}{|c|}{$(-4.4$ to 11.3$)$} \\
\hline \multicolumn{10}{|l|}{ Suicidal Ideation Questionnaire ${ }^{b}$} \\
\hline Group therapy & 32 & 89.1 & 44.4 & 28 & 41.3 & 39.6 & 28 & 47.3 & 50.5 \\
\hline Routine care & 28 & 83.9 & 51.1 & 29 & 46.0 & 48.9 & 27 & 39.7 & 46.7 \\
\hline Mean difference & \multirow{2}{*}{\multicolumn{3}{|c|}{$\begin{array}{c}5.2 \\
(-19.5 \text { to } 29.9)\end{array}$}} & \multirow{2}{*}{\multicolumn{3}{|c|}{$\begin{array}{c}-4.6 \\
(-28.3 \text { to } 19.1)\end{array}$}} & \multirow{2}{*}{\multicolumn{3}{|c|}{$(-18.8$ to 33.9$)$}} \\
\hline $95 \%$ CI of difference & & & & & & & & & \\
\hline \multicolumn{10}{|l|}{$\mathrm{HoNOSCA}^{c}$} \\
\hline Group therapy & 32 & 18.0 & 4.3 & 31 & 9.6 & 6.8 & 31 & 8.4 & 6.4 \\
\hline Routine care & 31 & 18.6 & 6.2 & 31 & 11.7 & 8.6 & 31 & 6.9 & 6.1 \\
\hline Mean difference & \multirow{2}{*}{\multicolumn{3}{|c|}{$\begin{array}{c}-0.6 \\
(-3.3 \text { to } 2.1)\end{array}$}} & \multirow{2}{*}{\multicolumn{3}{|c|}{$\begin{array}{c}-2.0 \\
(-6.0 \text { to } 1.9)\end{array}$}} & \multirow{2}{*}{\multicolumn{3}{|c|}{$\begin{array}{c}1.5 \\
(-1.7 \text { to } 4.7)\end{array}$}} \\
\hline $95 \%$ CI of difference & & & & & & & & & \\
\hline
\end{tabular}

Note: $\mathrm{CI}=$ confidence interval.

${ }^{a}$ Mood and Feelings Questionnaire scores range from 0 to 68, with high scores indicating greater depression.

${ }^{b}$ Suicidal Ideation Questionnaire (30-item version) scores range from 0 to 180 , with high scores indicating higher suicidal ideation.

${ }^{c}$ Health of the Nation Outcome Scales for Children and Adolescents (HoNOSCA) scores range from 0 to 52, with higher scores indicating greater problems.

multiple regression analysis conducted within the active treatment arm, more sessions of group therapy were associated with fewer incidents of deliberate self-harm $(B=$ $-0.06,95 \% \mathrm{CI}-0.1$ to $0.0, p<.05$; partial correlation $-0.36)$, even when the number of incidents of deliberate self-harm before randomization and total HoNOSCA score at baseline (as a measure of severity) were controlled. By contrast, the same analysis within the control group found that more sessions of routine care were associated with more incidents of deliberate self-harm $(B=$ $0.26,95 \%$ CI 0.11 to 0.41 ; partial correlation 0.56 ).

\section{DISCUSSION}

This preliminary study set out to examine the efficacy of a group intervention for adolescents who had repeatedly harmed themselves. As far as we know, this is the first published randomized trial of any form of therapy for repeated self-harm in this age group. The results are promising. Although the group therapy did not significantly reduce levels of depression or suicidal thinking, it did lead to a reduction in repetition of deliberate selfharm. There was a particularly strong effect of the group treatment on the risk of a second episode of self-harm, with an absolute risk reduction of $26 \%$. This means that we would have to treat just four adolescents (95\% CI for number needed to treat 3 to 14 ) with the group intervention (rather than routine care) for 6 months to prevent one additional adolescent from repeating self-harm on two or more occasions.

\section{Methodological Issues and Limitations}

When interpreting the results of this study, however, a number of issues should be borne in mind. First, when this study was devised and funded it was recognized that it could detect only a large effect of the group intervention on repetition of self-harm. The relatively small sample size meant that the confidence intervals for the size of the effect of the intervention were wide. For instance, although an estimate of number needed to treat of 4 sounds impressive, the upper $95 \%$ confidence interval for this estimate was 14 . It is therefore possible that 14 adolescents would have to be treated for 6 months with a median of eight treatment sessions per case in order to prevent just one adolescent from repeating on two or more occasions. Although some of these repetitions were serious, some were relatively mild, consisting perhaps of the deliberate ingestion of just a few analgesic tablets. Some service planners might feel that this is not an efficient use of resources. A larger study will be required to 
estimate more reliably the efficacy of the intervention in preventing repetition of deliberate self-harm.

Second, this study was conducted in just one health district by experienced therapists who had devised the intervention. It remains to be seen whether the intervention can be used successfully in other services. Third, this study did not assess costs and cannot therefore come to any meaningful conclusions about the cost-effectiveness of the intervention. Fourth, although our within-trial arm analyses of the relationships between numbers of sessions and outcome did attempt to control for potential confounders, it is possible those given more routine care were more disturbed than those given less. The association of amount of routine care and outcome could be spurious. Fifth, some caution is needed in interpreting the apparent effects of the intervention on behavioral disorders. This study had only a relatively brief follow-up, and it is possible that these effects were not sustained. Sixth, the diagnostic procedures used in this study meant that we relied to an important extent on the young person's account of their outcomes. Finally, the intervention did not alter global outcome or levels of depression.

\section{Comparisons With Previous Research}

Although it is difficult to make direct comparisons with research in older age groups, our results are consistent with this research. Thus, for instance, our finding of a beneficial effect of the intervention on repetition of self-harm but not on depression was also reported by Linehan and colleagues (1991).

There is some evidence that putting delinquent boys into therapeutic groups might worsen their difficulties (Dishion et al., 1999). In our study, however, the group intervention tended, if anything, to improve behavioral problems.

\section{Research Implications}

Our preliminary study shows that it is feasible to conduct a randomized trial with this challenging group of patients, to the extent that the majority of cases agreed to enter the study and most agreed to have some treatment. However, our results also show that very large studies will be needed to detect reliably an effect of treatment on some indicators of repetition of self-harm. For example, we estimate that to detect a 33\% reduction in the total number of episodes of repeated deliberate self-harm (which from a public health perspective is arguably the most important outcome), a sample size of approximately 150 cases in each arm would be required.

\section{Clinical Implications}

In previous randomized trials with depressed or suicidal adolescents, we have tested relatively brief forms of intervention (Harrington et al., 1998; Wood et al., 1996). The rationale for studying brief treatments was that if they worked, they could be easily implemented in routine clinical practice. Indeed, it is sometimes the case in psychotherapy outcomes research that "more is not always better" (Bickman, 1996). The results of the present research suggest, however, that effective prevention of repetition of deliberate self-harm may require that high-risk cases attend treatment for several months. More sessions of group therapy were associated with a better outcome, whereas more sessions of routine care were associated with a worse outcome. More of the right kind of treatment may sometimes be better.

\section{REFERENCES}

Ambrosini PJ (2000), Historical development and present status of the Schedule for Affective Disorders and Schizophrenia for School-Age Children (K-SADS). J Am Acad Child Adolesc Psychiatry 39:49-58

American Psychiatric Association (1994), Diagnostic and Statistical Manual of Mental Disorders, 4th edition (DSM-IV). Washington, DC: American Psychiatric Association

Angold A, Costello EJ, Pickles A, Winder F (1987), The development of a questionnaire for use in epidemiological studies of depression in children and adolescents. London: MRC Child Psychiatry Unit, Institute of Psychiatry

Bickman L (1996), A continuum of care: more is not always better. Am Psychol 51:689-701

Brown J, Cohen P, Johnson JG, Smailes EM (1999), Childhood abuse and neglect: specificity of effects on adolescent and young adult depression and suicidality. J Am Acad Child Adolesc Psychiatry 38:1490-1496

Byford S, Harrington RC, Torgerson D et al. (1999), Cost-effectiveness analysis of a home-based social work intervention for children and adolescents who have deliberately poisoned themselves: the results of a randomized controlled trial. Br J Psychiatry 174:56-62

Dishion TJ, McCord J, Poulin F (1999), When interventions harm: peer groups and problem behavior. Am Psychol 54:755-764

Fergusson DM, Horwood LJ, Lynskey MT (1996), Childhood sexual abuse and psychiatric disorder in young adulthood, II: psychiatric outcomes of childhood sexual abuse. J Am Acad Child Adolesc Psychiatry 35:1365-1374

Garralda ME, Yates P, Higginson I (2000), Child and adolescent mental health service use. HoNOSCA as an outcome measure. Br J Psychiatry 177:52-58

Gowers SG, Harrington RC, Whitton A et al. (1999a), Health of the Nation Outcome Scales for Children and Adolescents (HoNOSCA): glossary of the HoNOSCA score sheet. Br J Psychiatry 174:428-431

Gowers SG, Harrington RC, Whitton A et al. (1999b), Brief scale for measuring the outcomes of emotional and behavioural disorders in children: the Health of the Nation Outcome Scales for Children and Adolescents (HoNOSCA). Br J Psychiatry 174:413-416

Harrington RC, Fudge H, Rutter M, Pickles A, Hill J (1990), Adult outcomes of childhood and adolescent depression, I: psychiatric status. Arch Gen Psychiatry 47:465-473

Harrington RC, Kerfoot M, Dyer E et al. (1998), Randomized trial of a home based family intervention for children who have deliberately poisoned themselves. J Am Acad Child Adolesc Psychiatry 37:512-518

Hawton K, Arensman E, Wasserman D et al. (1998), Relation between attempted suicide and suicide rates among young people in Europe. $J$ Epidemiol Community Health 52:191-194 
Hawton K, Catalan J (1982), Attempted Suicide. Oxford, England: Oxford Medical Publications

Hawton K, Fagg J, Simkin S, Bale E, Bond A (2000), Deliberate self-harm in adolescents in Oxford, 1985-1995. J Adolesc 23:47-55

Hawton K, Houston K, Shepperd R (1999a), Suicide in young people: study of 174 cases, aged under 25 years, based on coroners' and medical records. Br J Psychiatry 175:271-276

Hawton K, Kingsbury S, Steinhardt K, James A, Fagg J (1999b), Repetition of deliberate self-harm by adolescents: the role of psychological factors. $J$ Adolesc 22:369-378

Hollis S, Campbell F (1999), What is meant by intention to treat analysis? Survey of published randomised controlled trials. Br Med J319:670-674

Kaufman J, Birmaher B, Brent D, Rao U, Ryan N (1996), Schedule for Affective Disorders and Schizophrenia for School Age Children (6-18 Years): Kiddie-SADS-Lifetime Version (K-SADS-PL), Version 1.0, October 1996. Pittsburgh: University of Pittsburgh

Kerfoot M (1984), Assessment of the young adolescent and the family. In: Suicide: Assessment and Intervention, Hatton CL, Valente SM, eds. Norwalk, CT: Appleton-Century-Crofts

Kerfoot M (1988), Deliberate self-poisoning in childhood and early adolescence. J Child Psychol Psychiatry 29:335-345

Kerfoot M, Dyer E, Harrington V, Woodham A, Harrington RC (1996), Correlates and short-term course of self-poisoning in adolescents. $\mathrm{Br} \mathrm{J}$ Psychiatry 168:38-42

Kerfoot M, Huxley P (1995), Suicide and deliberate self-harm in young people. Curr Opinion Psychiatry 8:214-217

Kerfoot M, McHugh B (1992), The outcome of childhood suicidal behaviour. Acta Paedopsychiatr 55:141-145
Kroll L, Harrington RC, Gowers S, Frazer J, Jayson D (1996), Continuation of cognitive-behavioral treatment in adolescent patients who have remitted from major depression: feasibility and comparison with historical controls. J Am Acad Child Adolesc Psychiatry 35:1156-1161

Linehan MM, Armstrong HE, Suarez A, Allmon D, Heard HL (1991), Cognitive-behavioral treatment of chronically parasuicidal borderline patients. Arch Gen Psychiatry 48:1060-1064

Machin D, Campbell MJ (1987), Statistical Tables for the Design of Clinical Trials. Oxford, England: Blackwell

Marks I (1981), Cure and Care of Neuroses. New York: Wiley

NHS Centre for Reviews and Dissemination (1998), Deliberate self-harm. Effective Health Care 4:1-12

Puig-Antich J, Chambers W (1978), The Schedule for Affective Disorders and Schizophrenia for School-Age Children. New York: New York State Psychiatric Institute

Reynolds WM (1988), Suicidal Ideation Questionnaire: Professional Manual. Odessa, FL: Psychological Assessment Resources

Sackett DL, Strauss SE, Richardson WS, Rosenberg W, Haynes RB (2000), Evidence-Based Medicine: How to Practice and Teach Evidence-Based Medicine, 2nd ed. Edinburgh, Scotland: Churchill Livingstone

Wood A, Kroll L, Moore A, Harrington RC (1995), Properties of the Mood and Feelings Questionnaire in adolescent psychiatric outpatients: a research note. J Child Psychol Psychiatry 36:327-334

Wood AJ, Harrington RC, Moore A (1996), Controlled trial of a brief cognitivebehavioural intervention in adolescent patients with depressive disorders. $J$ Child Psychol Psychiatry 37:737-746

World Health Organization (1996), Multiaxial Classification of Child and Adolescent Psychiatric Disorders. Cambridge, England: Cambridge University Press 